\title{
ALLOSTERIC MODELS FOR THE INTERACTION OF 2,3-DIPHOSPHOGLYCERIC ACID WITH HEMOGLOBIN
}

\author{
Lambert H. M. Janssen and Simon H. De Bruin \\ Department of Biophysical Chemistry, University of Nijmegen, \\ Toernooiveld, Nijmegen, The Netherlands
}

Received 3 July 1972

\begin{abstract}
The capabilities of the two state model of Monod, Wyman and Changeux and the sequential model of Koshland, Némethy and Filmer to explain the influence of 2,3diphosphoglyceric acid on the oxygen-binding properties of hemoglobin are compared. It appeared that, after introducing some modifications into the model of Koshland et al., the influence of 2,3-diphosphoglyceric acid on the oxygen-binding can better be described by this model than by the model of Monod et al. In addition, a method is presented, based entirely on oxygen-binding data of tetrameric hemoglobin, by which the parameters of the two state model can be evaluated.
\end{abstract}

Much is known about the structure of oxy- and deoxyhemoglobin, and a detailed stereochemical mechanism has been published by Perutz (1) to yplain the phenomenon of the heme-heme inter-ction. It has been shown that the two state model of Monod, Wyman \& Changeux (2) and the sequential model of Koshland, Némethy \& Filmer (3), further designated as the MWC and the KNF model, both are able to describe a given oxygenation curve satisfactorily.

Organic phosphates such as 2,3-diphosphoglyceric acid (DPG) are known to alter hemoglobin function substantially $(4,5)$. Chemical and crystallographic evidence points out that DPG is bound mainly to deoxyhemoglobin in a ratio $1: 1$, on the dyad axis, forming salt bridges with positive groups of only the $\beta$-chains $(1,6-9)$. Due to this binding the affinity of hemoglobin for oxygen is sharply reduced. In this paper it is our aim to describe in which manner the two mentioned allosteric models can explain this influence of DPG.

Because for this purpose a procedure for the determination of the parameters of the MWC model is required, we will start with a presenta- tion of this. As will be discussed elsewhere, the procedure proposed by Edelstein (10) is not correct.

Determination of the parameters of the MWC model

If $\mathrm{Y}$ is the fractional saturation of hemoglobin with oxygen and $p$ its partial pressure, then oxygen-binding data around $Y=0.5$ can reasonably be described by the Hill equation $\mathrm{Y}=$ $K \mathrm{p}^{\mathrm{n}} / 1+\mathrm{Kp}^{\mathrm{n}}$ where $\mathrm{K}$ is a constant. A plot of $\log (Y / 1-Y)$ versus $\log p$ is called the Hill plot and its slope $\mathrm{n}$ in the middle portion of saturation varies mostly between 2.5 and 2.9 , at least for normal hemoglobins; the value of the Hill parameter $\mathbf{n}$ is a measure for the heme-heme interaction (11-13). Wyman $(11,12)$ has argued that when $\mathbf{Y}$ approaches 0 or $1, \mathrm{n}$ should be 1 . Furthermore, he has demonstrated that from the distance between the two asymptotes in a Hill plot, the interaction energy $\Delta \mathrm{F}_{\mathrm{I}}$ per oxygen-binding site can be calculated.

In the Adair model (14) the total oxygenation curve is described by four successive oxygenation 
constants. In this scheme the fractional saturation takes the form

$Y=\frac{k_{1} p+3 k_{1} k_{2} p^{2}+3 k_{1} k_{2} k_{3} p^{3}+k_{1} k_{2} k_{3} k_{4} p^{4}}{1+4 k_{1} p+6 k_{1} k_{2} p^{2}+4 k_{1} k_{2} k_{3} p^{3}+k_{1} k_{2} k_{3} k_{4} p^{4}}(1)$

$k_{1}, k_{2}, k_{3}$ and $k_{4}$ being the intrinsic association constants for the reactions $\mathrm{Hb}\left(\mathrm{O}_{2}\right)_{1-1}+\mathrm{O}_{2} \rightleftharpoons$ $\mathrm{H}_{\mathrm{b}}\left(\mathrm{O}_{2}\right)_{\mathrm{i}}(\mathrm{i}=1$ to 4$)$.

Tyuma et al. (15) have shown that the following relations will hold:

$$
\begin{aligned}
& \log (\mathrm{Y} / 1-\mathrm{Y})=\log \mathrm{p}+\log \mathrm{k}_{\mathbf{1}} \text { as } \mathrm{p} \rightarrow 0 \\
& \log (\mathrm{Y} / 1-\mathrm{Y})=\log \mathrm{p}+\log \mathrm{k}_{\mathbf{4}} \text { as } \mathrm{p} \rightarrow \infty
\end{aligned}
$$

The Adair equation therefore predicts a unit slope for the Hill plot at low and high degree of saturation. It will be clear that in terms of the Adair model $\Delta \mathrm{F}_{\mathrm{I}}$ is equal to $-\mathrm{RT} \ln \left(\mathrm{k}_{1} / \mathrm{k}_{4}\right)$. (The term "interacting energy" used here stems from Wyman but it should be recognized that it does not represent the true interaction energy, which should be defined in another way [see SAROFF, H. A. \& Minton, A. P. (1972) Science 175, 1253-1255].

The MWC model is based on the assumption that an allosteric protein can exist in two states, called the $R$ and the $T$ state. The equilibrium between these two states in absence of ligand is ruled by the equilibrium constant $\mathrm{L}$. The ligand affinity of the $R$ state is described by the dissociation constant $K_{R}$, that of the $T$ state by $K_{T}$, with $\mathbf{K}_{\mathbf{R}} / \mathbf{K}_{\mathbf{T}}<1$.

$Y$ is given by

$$
\mathrm{Y}=\frac{\mathrm{Lc} \alpha(1+\mathrm{c} \alpha)^{3}+\alpha(1+\alpha)^{3}}{\mathrm{~L}(1+\mathrm{c} \alpha)^{4}+(1+\alpha)^{4}}
$$

where $\mathrm{c}=\mathrm{K}_{\mathrm{R}} / \mathrm{K}_{\mathrm{T}}$ and $\alpha=\mathrm{p} / \mathrm{K}_{\mathrm{R}}$ (2). It can be proven that, under certain restrictions which will be mentioned later, one can write

$$
\begin{array}{r}
\log (\mathrm{Y} / 1-\mathrm{Y})=\log \alpha \mathrm{c}=\log \mathrm{p}-\log \mathrm{K}_{\mathrm{T}} \\
\text { as } \alpha \rightarrow 0 \\
\log (\mathrm{Y} / 1-\mathrm{Y})=\log \alpha=\log \mathrm{p}-\log \mathrm{K}_{\mathrm{R}} \\
\text { as } \alpha \rightarrow \infty
\end{array}
$$

Therefore, from a Hill plot, $K_{R}$ and $K_{T}$ can be determined unambiguously provided that the measurements are extended to such $p$ values that the slope of the plot becomes unity. The allosteric constant $\mathbf{L}$ may be obtained by trial and error or by the relation

$$
\mathrm{L}=\left(\mathrm{p}_{\mathrm{m}} / \mathrm{K}_{\mathrm{R}}\right)^{4}
$$

with $\mathrm{p}_{\mathrm{m}}$ the median oxygen pressure (for defini- tion see Ref. 12). This relation can be derived from Equation 4 in the case $\operatorname{Lc}^{4}\langle\langle 1$. (The meaning of this condition can easily be understood if it is taken into consideration that $T_{n} / R_{n}=L c^{n}$, with $T_{n}\left(R_{n}\right)$ being the number of molecules in the T (R) state having $n(n=0$ to 4$)$ molecules of ligand bound.) If the curve $Y$ versus $\log p$ is symmetrical around $\mathrm{Y}=\mathbf{0 . 5}$ then $\mathrm{p}_{\mathrm{m}}=\mathrm{p}_{\mathrm{L} / 2}$, where $p_{1 / 2}$ is the oxygen pressure at half saturation. Furthermore, $\Delta F_{I}$ is now related to $K_{R}$ and $\mathrm{K}_{\mathrm{T}}$ by the equation $\Delta \mathrm{F}_{\mathrm{I}}=-\mathrm{RT} \ln \left(\mathrm{K}_{\mathrm{R}} / \mathrm{K}_{\mathrm{T}}\right)$ -RTlnc.

In the KNF model it is assumed that the individual subunits of an allosteric protein can exist either in a non-binding conformation $\mathbf{A}$ or a ligand-binding conformation $B$. The cooperativity is interpreted as being caused by the fact that the $B$ conformation gives rise to more favorable intrachain interactions than the A conformation.

In the KNF model there are four constants, $\mathrm{K}_{\mathrm{All}}$ and $\mathrm{K}_{\mathrm{BB}}$, which define interactions between subunits; $\mathrm{K}_{\mathrm{S}}$, a substrate-binding constant; and $\mathrm{K}_{\mathrm{t}}$ describing the equilibrium between the $\mathrm{A}$ and $B$ conformation. The reader is referred to the original paper for the description of the methods by which the several constants can be determined (3).

In principle, the values of $K_{R}, K_{T}$ and $L$ may be obtained from Equations 5, 6 and 7. However, if the Adair constants describing an oxygen saturation curve have been determined beforehand, we may take advantage of these data. Tr MWC binding Equation 4 is written in the for... of the Adair Equation 1. The result is shown in the second column of Table 1. This means that if these values for $k_{1}$ to $k_{4}$ are substituted in Equation 1, the original binding Equation 4 is obtained again. The same can be done for the equation giving the fractional saturation of the tetrahedral KNF model (Equation 13 of Ref. 3). The resulting equations are shown in the third column of Table 1. From this Table it follows that

$$
\mathrm{k}_{1}=1 / \mathrm{K}_{\mathrm{T}}
$$

provided that $(1+\mathrm{Lc}) /(1+\mathrm{L}) \approx \mathrm{c}$, and

$$
\mathbf{k}_{4}=1 / \mathbf{K}_{\mathbf{R}}
$$

provided that $\left(1+\mathbf{L c}^{4}\right) /\left(1+\mathbf{L} c^{3}\right) \approx 1$. It should be noted that $k_{1}$ and $k_{4}$ are defined as association constants and $\mathrm{K}_{\mathbf{R}}$ and $\mathrm{K}_{\mathbf{T}}$ as dissociation constants. Hopfield, Shulman \& Ogawa (16) discussing the kinetic aspects of the MWC model arrived also at Equation 8 and 9. If the condi- 
TABLE 1

Relation between the Adair, the MWC and the (tetrahedral) KNF model

(The modified KNF model as described in the text has been used to fit oxygen-binding curves in the presence of DPG. Note that when $\mathrm{K}_{\mathrm{A}^{\prime} \mathrm{A}^{\prime}}=1$, which means that there is no preference for (AA) or ( $\mathrm{A}^{\prime} \mathrm{A}^{\prime}$ ) interaction, the modified KNF model changes into the model used in the absence of DPG.)

\begin{tabular}{|c|c|c|c|c|c|}
\hline Adair & & MWC & & KNF & Modified KNF \\
\hline $\mathbf{k}_{1}$ & $\frac{1}{K_{R}}$ & $\frac{1+L c}{1+L}$ & $\frac{1}{K_{T}}$ & $\mathrm{~K}_{\mathrm{AB}}^{3} \mathrm{~K}_{\mathbf{8}} \mathrm{K}_{\mathrm{t}}$ & $\begin{array}{l}1 \\
-\frac{1}{2} \\
\mathbf{K}_{\mathbf{A B}}^{\mathbf{s}}\left(1+1 / \mathbf{K}_{\mathbf{A}^{\prime} \mathbf{A}^{\prime}}\right) \mathbf{K}_{\mathbf{s}} \mathbf{K}_{\mathrm{t}}\end{array}$ \\
\hline $\mathrm{k}_{2}$ & $\frac{1}{\mathbf{K}_{\mathbf{R}}}$ & $\frac{1+L c^{2}}{1+L c}$ & & 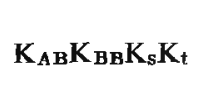 & $\frac{1}{3} \times \frac{\mathbf{K}_{\mathrm{AB}} \mathbf{K}_{\mathrm{BB}}\left(\mathrm{K}_{\mathrm{A}^{\prime} \mathrm{A}^{\prime}}+5\right)}{\mathbf{K}_{\mathrm{A}^{\prime} \mathrm{A}^{\prime}}+1} \mathbf{K}_{\mathrm{s}} \mathbf{K}_{\mathbf{t}}$ \\
\hline $\mathrm{k}_{\mathrm{a}}$ & $\frac{1}{K_{R}}$ & $\frac{1+\mathbf{L c}^{3}}{1+\mathbf{L c}^{2}}$ & & $\mathbf{K}_{\mathbf{A B}}^{-1} \mathbf{K}_{\mathbf{B B}}^{2} \mathbf{K}_{\mathbf{g}} \mathbf{K}_{t}$ & $6 \times \frac{K_{A B}^{-1} K_{B B}^{2}}{K_{A^{\prime} A^{\prime}}^{2}+5} K_{s} K_{t}$ \\
\hline $\mathrm{k}_{4}$ & $\frac{1}{\mathbf{K}_{\mathbf{R}}}$ & $\frac{1+\mathbf{L c}^{4}}{1+\mathbf{L c}^{3}}$ & $\frac{1}{\mathrm{~K}_{\mathbf{R}}}$ & $\mathrm{K}_{\mathrm{A} B}^{-3} \mathrm{~K}_{\mathrm{tB} B}^{3} \mathrm{~K}_{\mathrm{S}} \mathrm{K}_{\mathrm{t}}$ & $\mathrm{K}_{\mathrm{AB}}^{-3} \mathrm{~K}_{\mathrm{BB}}^{3} \mathrm{~K}_{\mathrm{S}} \mathrm{K}_{\mathrm{t}}$ \\
\hline
\end{tabular}

tions for using these equations are not totally fulfilled, they can still yield approximate values which may be used for an iterative procedure in order to obtain better values.

If an oxygenation curve is known in terms of the Adair constants one can furthermore show that

$$
\mathrm{p}_{\mathrm{m}}^{4}=\left(\mathrm{k}_{1} \mathrm{k}_{2} \mathrm{k}_{3} \mathrm{k}_{4}\right)^{-1}
$$

- nis equation, combined with Equations 7 and 9, gives a direct relation between $L$ and the Adair constants, viz.

$$
\mathbf{L}=\mathbf{k}_{4}^{8} /\left(\mathbf{k}_{1} \mathbf{k}_{2} \mathbf{k}_{3}\right)
$$

Table 2 shows values of the parameters of the MWC model obtained as described above. From the data listed it can be confirmed that $(1+\mathrm{Lc})$ / $(1+\mathrm{L})=1.01 \mathrm{c}$ and $\left(1+\mathrm{Lc} c^{4}\right) /\left(1+\mathrm{Lc}^{3}\right)=0.97$. Therefore, the relations (8) and (9) hold true within a few percent. Following the procedure outlined by Koshland et al. (3) we found for the same set of oxygenation data $\mathrm{K}_{\mathrm{AB}}=1.0, \mathrm{~K}_{\mathrm{BB}}=$ 4.1 and $K_{8} K_{t}=0.063$.

In Table 3 the corresponding values for the Adair constants predicted by the MWC and KNF model are listed. It will be clear that both models are able to fit an oxygenation curve, although the correspondance is even better for the KNF model. It is worthwhile to mention here that Monod et al. (2) have reported $\mathrm{L}=9 \times 10^{3}$ and $\mathrm{c}=0.014$ (horse hemoglobin, $\mathrm{pH}$ 7).

\section{Interaction of DPG with hemoglobin}

DPG decreases the oxygen affinity of hemoglobin but has no drastic influence on the cooperativity. It binds only to deoxyhemoglobin (6). For the MWC model we therefore expect that, due to the binding of DPG, the $T$ conformation is favored resulting in an increase of $\mathrm{L}$. In the absence of DPG we estimated $L=4.7 \times 10^{3}$, and because

TABLE 2

Values of allosteric constants for the $M W C$ model

(The values of the Adair constants $\mathrm{k}_{1}$ and $\mathrm{k}_{4}\left(\mathrm{mmHg}^{-1}\right), \mathrm{p}_{1 / 2}(\mathrm{mmHg})$ and $\mathrm{n}$ have been taken from Tyuma et al.

(15) (human hemoglobin, $25^{\circ}, \mathrm{pH} 7.4$ ).)

\begin{tabular}{rlllllllll} 
Hemoglobin . . & $\mathrm{k}_{1}$ & $\mathrm{k}_{4}$ & $\mathrm{n}$ & $\mathrm{p}_{1 / 2}$ & $\mathrm{p}_{\mathrm{m}}$ & $\mathrm{K}_{\mathrm{R}}$ & $\mathrm{K}_{\mathrm{T}}$ & $\mathrm{c}$ & $\mathrm{L}$ \\
Stripped. . . . . & 0.079 & 4.35 & 2.5 & 1.9 & 1.9 & 0.23 & 12.7 & 0.018 & $4.7 \times 10^{3}$ \\
+ DPG. . . . . & 0.008 & 4.35 & 3.0 & 15.3 & 14.0 & 0.21 & 125 & 0.0017 & $2 \times 10^{7}$ \\
\hline
\end{tabular}


TABLE 3

Comparison of the $M W C$ and the KNF model

(The values of the Adair constants (in $\mathrm{mmHg}^{-1}$ ) were taken from Tyuma et al. (15). The constants for the MWC and the KNF model have been calculated as described in the text.)

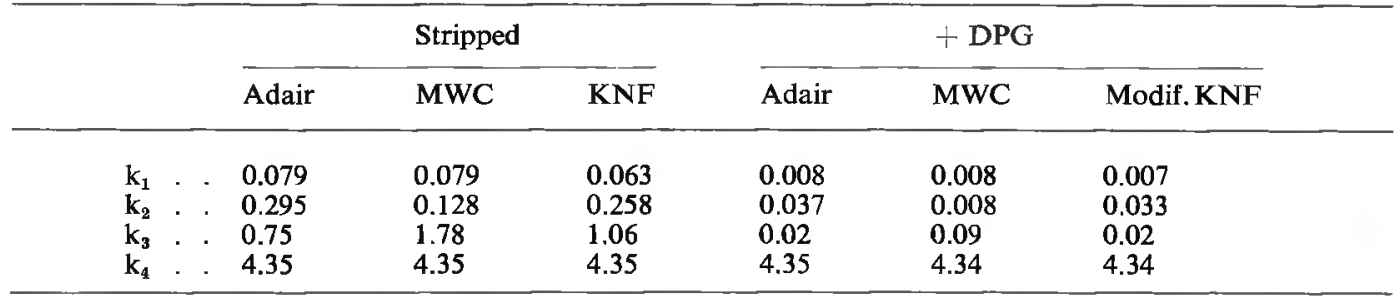

the association constant for the hemoglobin-DPG complex is near $10^{5}(6,17)$, we expected $\mathrm{L}$ to be near $5 \times 10^{8}$ in the presence of DPG. The observation that no DPG is bound by oxyhemoglobin very probably means that $\mathbf{K}_{\mathbf{R}}$ will not change by the addition of DPG. These facts basically explain the lower oxygen affinity of hemoglobin in the presence of DPG (see Equation 7). On the other hand if this were the only effect of DPG we would expect $\mathbf{n}$ to be near 1 (see Fig. 1) in contrast with the experimental results. Therefore, by the presence of DPG, c should decrease and, therefore, $\mathrm{K}_{\mathrm{T}}$ should increase. The Adair constants given by Tyuma et al. (15) (see Table 3) are in agreement with these predictions. Analyzing those constants according to the principles outlined above we obtain the parameters listed in

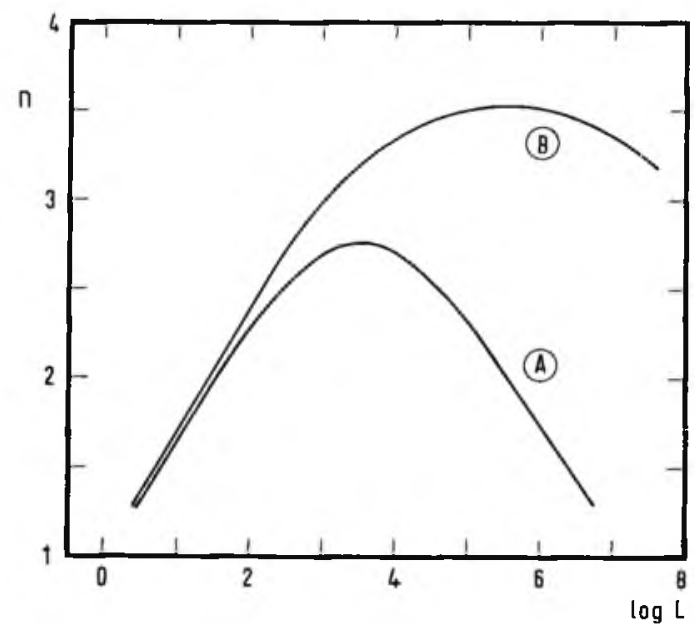

FIGURE 1

Dependence of Hill parameter $n$ on $L$ and $c$. Curve A has been calculated using $\mathrm{c}=\mathbf{0 . 0 1 8}$, curve $\mathrm{B}$ using $c=0.0017$.
Table 2 and the Adair constants according to the MWC model listed in Table 3. The fitting of the experimental curve as judged by comparison of the Adair constants is, however, fairly poor. Moreover, the Hill parameter calculated from these constants is 3.5 (see Fig. 1) and is much higher than the observed value of 3.0.

The KNF model, as used above to explain the oxygen-binding data of stripped hemoglobin, also gives a poor fit of the data. But, in this model we can account for the specific interactions of DPG. Since DPG binds only to the $\beta$-chains of hemoglobin thereby constraining it in the deoxy form or, in terms of the KNF model, in the A form, it might be expected that in the presence of DPG the interaction constant $K_{A A}$ between two $\beta$-chains is different from that between two $\alpha$-chains or between the $\alpha$ - and the $\beta$-chains. $\mathrm{W}^{\prime}$ therefore introduce a constant $K_{A^{\prime} A^{\prime}}$ defined $b_{y}$

$$
K_{\mathbf{A}^{\prime} \mathbf{A}^{\prime}}=\frac{\left(\mathrm{A}^{\prime} \mathbf{A}^{\prime}\right)(\mathrm{A})(\mathrm{A})}{(\mathbf{A} \mathbf{A})\left(\mathbf{A}^{\prime}\right)\left(\mathbf{A}^{\prime}\right)}
$$

where, following the KNF notation, $\left(A^{\prime} A^{\prime}\right)$ and (AA) refer to interacting pairs and (A') and (A) to non-interacting subunits. Therefore, $K_{\mathbf{A}^{\prime} \mathbf{A}^{\prime}}$ is a measure for the interaction energy of $\left(A^{\prime} A^{\prime}\right)$ relative to the interaction energy of (AA). We expect that $\left.K_{A^{\prime} A^{\prime}}\right)>1$ which means that $\left(A^{\prime} A^{\prime}\right)$ interaction is more favorable than (AA) interaction. We will assume that, only when both $\beta$-chains are in the $\mathbf{A}$ conformation, would the parameter $K_{A^{\prime} A^{\prime}}$ be introduced, thus accounting for the interaction between these particular chains. This means that we assume that DPG is released when one of the $\beta$-chains becomes liganded. All other interactions are taken to be similar to those for stripped hemoglobin. The concentration of the several species (using the KNF notation) is given by Equations 13-16. 
$A_{3} B S=\left(2 K_{A B}^{a}+2 K_{A^{\prime} A^{\prime}}^{-1} K_{A B}^{3}\right)\left(K_{9} K_{t}\right)\left(A_{4}\right)$

$A_{2} B_{2} S_{2}=\left(K_{A B}^{4} K_{B B}+5 K_{A^{\prime} A^{\prime}}^{-1} K_{A B}^{4} K_{B B}\right)\left(K_{6} K_{t}\right)^{2}$ (A)

$$
\begin{gathered}
\mathrm{AB}_{3} \mathrm{~S}_{3}=4 \mathrm{~K}_{\mathrm{A}^{\prime} \mathrm{A}^{\prime}}^{-1} \mathrm{~K}_{\mathrm{AB}}^{3} \mathrm{~K}_{\mathrm{BB}}^{3}\left(\mathrm{~K}_{\mathrm{8}} \mathrm{K}_{\mathrm{t}}\right)^{8}\left(\mathrm{~A}_{4}\right) \\
\left.\mathbf{B}_{4} \mathrm{~S}_{4}=\mathrm{K}_{\mathrm{A}^{\prime} \mathrm{A}^{\prime}}^{-1} \mathrm{~K}_{\mathrm{BB}}^{6} \mathrm{~K}_{\mathrm{S}} \mathrm{K}_{\mathrm{t}}\right)^{4}\left(\mathrm{~A}_{4}\right)
\end{gathered}
$$

The resulting binding equation, expressed as Adair constants, has been listed in the last column of Table 1.

In order to see if this model could account for the observed Adair constants, it should be a prerequisite that $\mathrm{K}_{\mathrm{s}}$ and $\mathrm{K}_{t}$, because they are intrinsic constants, have the same values as in the absence of DPG. Basically the constants $\mathrm{K}_{\mathrm{AB}}$ and $K_{\mathrm{BB}}$ should also have the same values as found for stripped hemoglobin, but because of the binding of DPG, small distortions may occur which are likely to change these interactions somewhat. So using the value of 0.063 for $\mathrm{K}_{\mathrm{s}} \mathrm{K}_{\mathrm{t}}$, we found that the experimental Adair constants could very well be simulated using $\mathrm{K}_{\mathrm{A}^{\prime} \mathrm{A}^{\prime}}=190, \mathrm{~K}_{\mathrm{AB}}=$ 0.61 and $K_{\mathrm{BB}}=2.5$ (see Table 3). Also very striking is the fact that this extended model is able to account for the ratio $k_{3} / k_{2}<1$. The much better correspondence obtained with this model between observed and calculated Adair constants seems to justify our assumption that DPG is released upon oxygen-binding by one of the $\beta$ chains. Furthermore, according to this modified KNF model, the first and second molecules of oxygen are mainly bound to the $\alpha$-chains (to an extent of more than $97 \%$ ). So this model, which gives a very good explanation for the Adair constants in the presence of DPG, implies that the $a$-chains are oxygenated first and that DPG is released when the third molecule of oxygen is bound, in analogy with the ideas proposed by Perutz (1).

Comparing the Adair constants of the MWC and the KNF model in Table 3 it will be evident that, at least in the presence of DPG, the details of the fitting are better described by the (modified) KNF than by the MWC model. This conclusion depends partially on the reliability of the figures used. However, Gibson (18) has reported kinetic constants for the dissociation of oxygen from liganded stripped and non-stripped hemoglobin.
The Adair constants calculated from his data of non-stripped hemoglobin show a nearly tenfold decrease of $k_{1}$, a ratio $k_{3} / k_{2}$ smaller than one, and $a \mathbf{k}_{\mathbf{4}}$ value unchanged as compared with stripped hemoglobin, these being similar to reports from Tyuma et al. (15). The absolute values of the Adair constants differ because of the different experimental conditions. Finally, we want to remark that the way in which the modified KNF model explains the effect of DPG is easier to understand and more in agreement with other experiments than that of the MWC model.

\section{REFERENCES}

1. Perutz, M. F. (1970) Nature 228, 726-739.

2. Monod, J., Wyman, J. \& Changeux, J.-P. (1965) J. Mol. Biol. 12, 88-118.

3. Koshland, D. E., Némethy, G. \& Filmer, D. (1966) Biochemistry 5, 365-385.

4. BeNESCH, R. \& BENESCH, R. E. (1967) Biochem. Biophys. Res. Commun. 26, 162-167.

5. Chanutin A. \& Curnish, R. R. (1967) Arch. Biochem. Biophys. 121, 97-102.

6. BentesCH, R., BenesCh, R. E. \& Yu, C. I., (1968) Proc. Nat. Acad. Sci. U.S. 59, 526-532.

7. De Verdier, C. H. \& GaRBY, L. (1969) Scand. J. Clin. Lab. Invest. 23, 149-151.

8. BunN, H. F. \& BrieHL, R. W. (1970) J. Clin. Invest. 49, 1088-1095.

9. ARNONE, A. (1972) Nature 237, 146-149.

10. EDELSTEIN, S. J. (1971) Nature 230, 224-227.

11. Wrman, J. (1948) Advan. Protein Chem. 4, 407531.

12. Wyman, J. (1964) Advan. Protein Chem., 19, 223286.

13. Wyman, J. (1967) J. Am. Chem. Soc. 89, 22022218.

14. Adair, G. S. (1925) J. Biol. Chem., 63, 529-545.

15. Tyuma, I., Shimizu, K. \& ImaI, K. (1971) Biochem. Biophys. Res. Commun. 43, 423-428.

16. Hopfield, J. J., Shulman, R. G. \& Ogawa, S. (1971) J. Mol. Biol. 61, 425-443.

17. De Bruin, S. H., Janssen, L. H. M. \& Van Os, G. A. J. (1971) Biochem. Biophys. Res. Commun. $45,544-550$.

18. Gibson, Q. H. (1970) J. Biol. Chem. 245, 32853288.

Address:

Lambert H. M. Janssen

Department of Biophysical Chemistry

University of Nijmegen

Toernooiveld, Nijmegen

The Netherlands 